\title{
A 1-V recycling current OTA with improved gain-bandwidth and input/output range
}

\author{
Xiao Zhao ${ }^{1,2}$, Qisheng Zhang ${ }^{1,2 a)}$, and Ming Deng ${ }^{1,2}$ \\ ${ }^{1}$ Key Laboratory of Geo-detection (China University of Geosciences, Beijing), \\ Ministry of Education \\ ${ }^{2}$ School of Geophysics and Information Technology, China University of \\ Geosciences (Beijing), Beijing 100083, P.R. China \\ a) zqs@cugb.edu.cn
}

Abstract: A novel bulk-driven technique is adopted to improve the gain-bandwidth of the conventional recycling current OTA, without requiring any additional power dissipation. Also, the threshold voltage of the transistors is reduced because of bulk-biasing technique, leading to the input/output voltage range extended. To compare the performance advantages of the proposed OTA versus the conventional one, two OTAs were designed in a $1-\mathrm{V} 0.18 \mu \mathrm{m}$ CMOS process. Results show that the proposed OTA's unit-gain bandwidth is improved by $55 \%$ and the input/output voltage range is increased by $140 / 110 \mathrm{mV}$. Also, the dc gain is enhanced almost $6 \mathrm{~dB}$.

Keywords: bulk-biasing technique, bulk-driven technique, gainbandwidth improvement, recycling current OTA

Classification: Integrated circuits

\section{References}

[1] G. Geelen, E. Paulus, D. Simanjuntak, H. Pastoor and R. Verlinden: IEEE ISSCC Dig. Tech. Papers (2006) 214.

[2] R. S. Assaad and J. Silva-Martinez: IEEE J. Solid-State Circuits 44 (2009) 2535.

[3] X. Zhao, H. Fang and J. Xu: Analog Inte. Cir. and Sig. Processing 71 (2012) 259.

[4] T.-H. Lin, C.-K. Wu and M.-C. Tsai: IEEE Trans. Circuits Syst. II, Exp. Briefs 54 (2007) 131.

[5] S. Chatterjee, Y. Tsividis and P. Kinget: Proc. 30th ESSCIRC (2004) 147.

[6] M. Taherzadeh-Sani and A. A. Hamoui: IEEE J. Solid-State Circuits 46 (2011) 660 .

\section{Introduction}

Operational transconductance amplifiers (OTAs) are important building 
blocks for analog circuits and systems [1]. In recent years, the recycling current folded cascode (RFC) amplifier is widely used because of its improved gain-bandwidth (GBW) and dc gain [2]. However, the increasing demands in portable and biomedical applications require amplifiers of lower power consumption and lower supply voltage [3].

In an OTA, the ratio of transconductance to current consumption reflects the power efficiency. In order to obtain lower power, the efficiency of the OTA must be improved $[4,5]$. In addition, with the scaling of the supply voltages, the reduction of the threshold voltage is not aggressive, which limits signal swings [6]. As a result, to extend signal swings of an OTA is necessary.

In this paper, a proposed bulk-driven recycling current folded cascode OTA (BDRFC) is presented. The bulk-driven technique is adopted to improve its gain-bandwidth without requiring any additional power. Also, the bulk-biasing technique is used to reduce the threshold voltage of the transistors, to extend the input/output voltage range. This brief is organized as follows. In Section 2, the architecture of the proposed OTA is introduced and the detailed circuit analysis is discussed. The circuit performance is next presented in Section 3, with conclusions given in Section 4.

\section{The proposed BDRFC OTA}

\subsection{Basic topology}

The conventional recycling current folded cascode OTA (RFC) is shown in Fig. 1. The input differential pairs are split in half $(M 1 a, b$ and $M 2 a, b)$ and the cross-over current mirrors (M3 : $M 4$ and $M 5: M 6)$ ensure that the

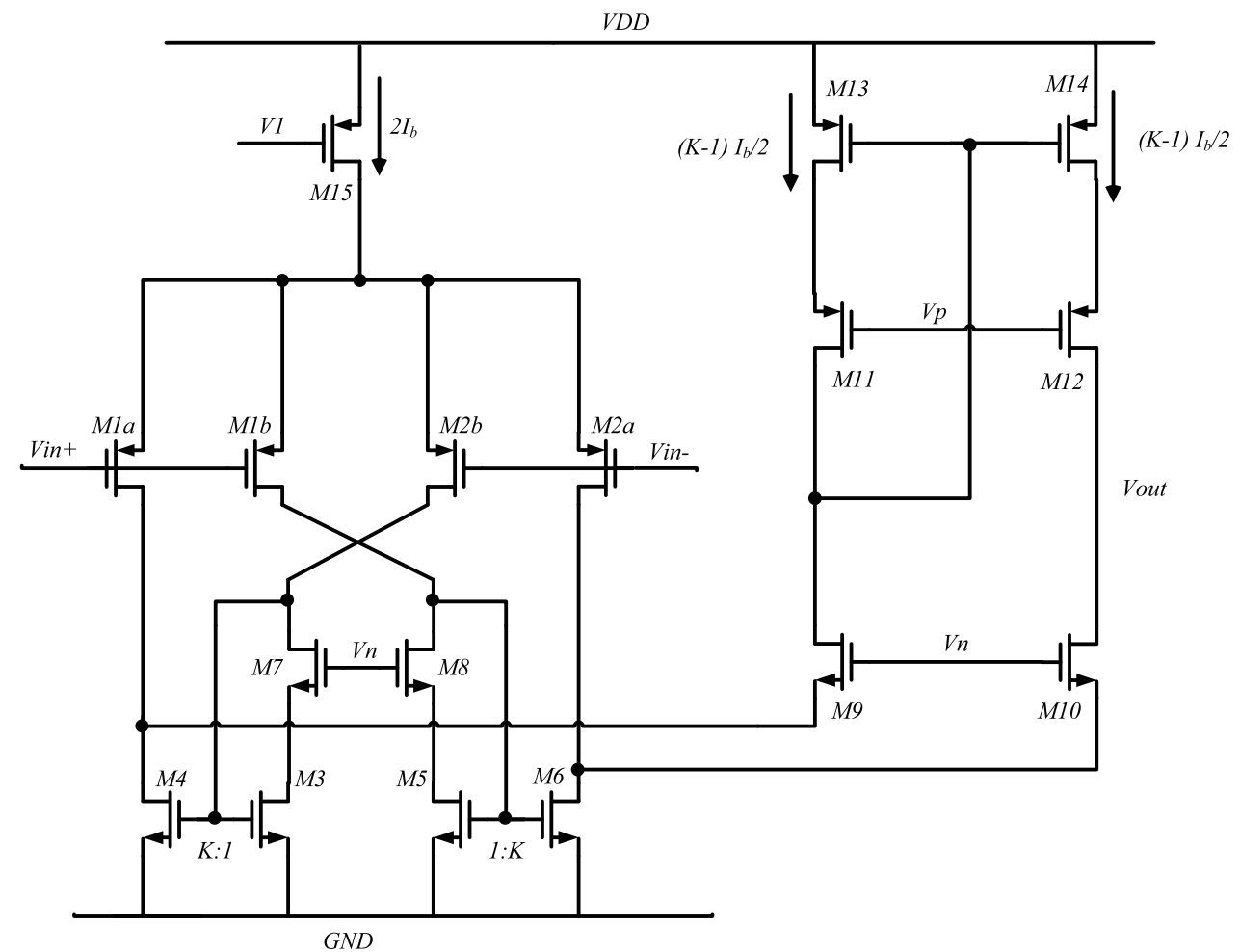

Fig. 1. Conventional recycling current folded cascode OTA (RFC) 


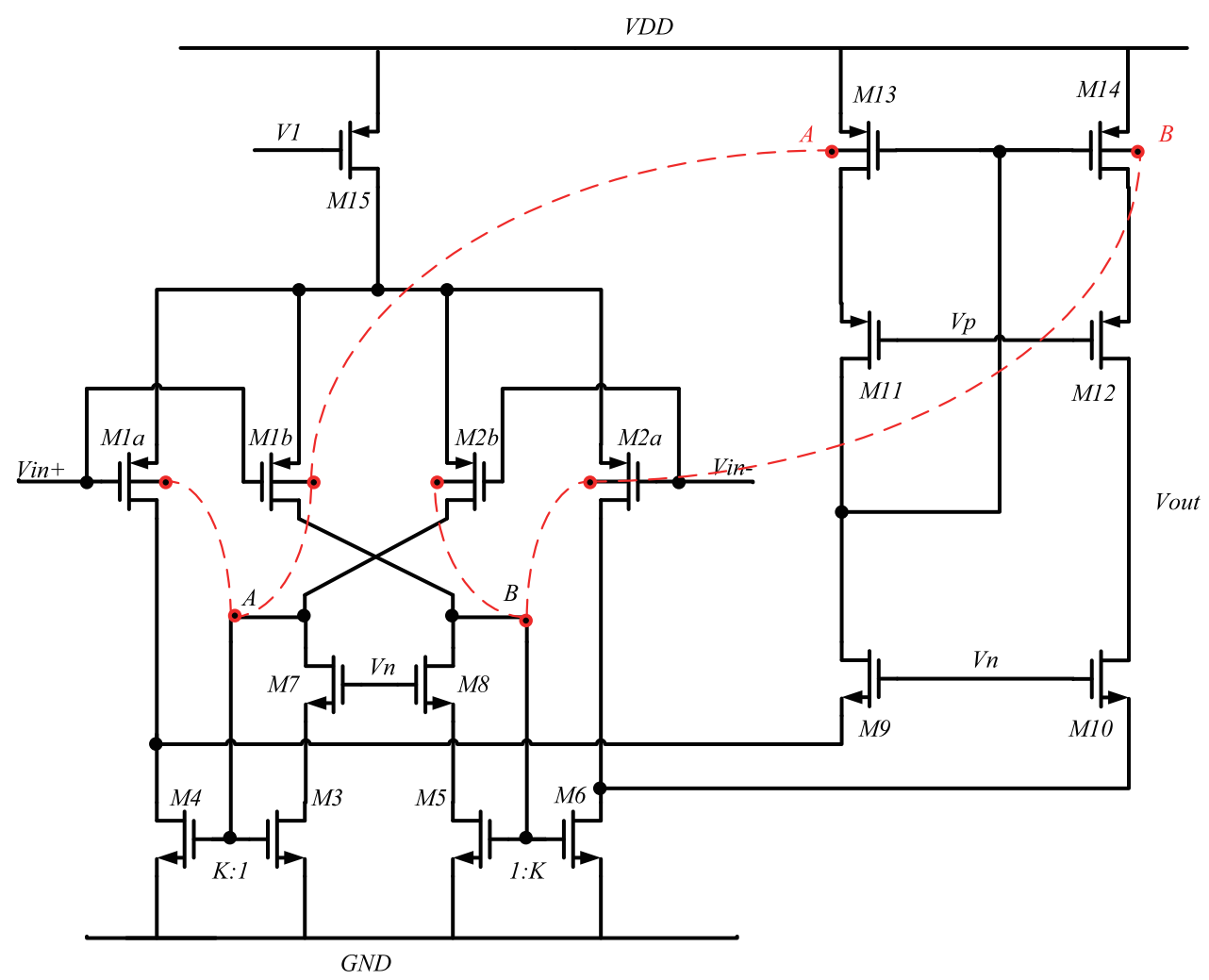

Fig. 2. Architecture of the proposed BDRFC OTA

effective transconductance $\left(G_{m}\right)$ of the RFC is improved by a ratio factor $K$, which leads to boost in the unity-gain bandwidth (GBW) and dc gain.

In order to further improve the GBW of the RFC, the bulk-driven technique is proposed to improve its transconductance. Also, the bulk-biasing technique is used to extend the input/output common-mode voltage range of the conventional RFC. The architecture of the proposed BDRFC OTA is shown in Fig. 2.

\subsection{Unity-gain bandwidth enhancement}

In bulk-driven technique, if the gate terminal is biased properly to turn on the MOS, the signal can be applied between the bulk and the source junctions so that the drain-to-source current can be modulated. Thereby a bulk terminal can behave as a second gate.

In the conventional RFC, the bulk terminal can be used to conduct the small signal current, thereby we apply the gate of the cross-over current mirror (at nodes A and B) to drive the bulk terminals of input pairs Mia,b $(\mathrm{i}=1-2)$, as shown in the red dashed lines of Fig. 2. At the same time, the bulk of the current mirror load (M13: M14) can also be used to drive the input small signal current, as shown in the red dashed lines of Fig. 2. The bulk transconductance $\left(g_{m b}\right)$ of both the input pairs and the current mirror load contribute to the overall transconductance $\left(G_{m}\right)$ and thereby increases the unity-gain bandwidth (GBW).

The bulk transconductance $\left(g_{m b}\right)$ is expressed as,

$$
g_{m b}=\eta g_{m}=\frac{d i_{D}}{d v_{B S}}=\frac{\gamma g_{m}}{2 \sqrt{2 \phi_{F}-V_{B S}}}
$$


reportedly, if the bulk-to-source junction is not forward biased, the ratio $\eta$ ranges from 0.2 to 0.4 , depending on the bulk-to-source voltage $\left(V_{B S}\right)$ and other specific process parameters.

In the conventional RFC, the effective transconductance $\left(G_{m}\right)$ is expressed as,

$$
G_{m R F C} \simeq(K+1) \cdot g_{m 1 b}
$$

From Fig. 2, it can be seen that the effective transconductance $\left(G_{m}\right)$ of the proposed BDRFC is expressed as,

$$
G_{m B D R F C} \simeq\left[\left(\frac{g_{m b 1 b}}{g_{m 5}}+1\right) \cdot(K+1)+\frac{g_{m b 14}}{g_{m 5}}\right] \cdot g_{m 1 b}
$$

where $g_{m i}$ and $g_{m b i}$ is the transconductance and bulk transconductance of transistor $M_{i}$.

When the factor $K$ is equal to 3 , the bias current $\left(I_{B}\right)$ in $M_{1 b}$ is the same as that in $M_{5}$, and $I_{B}$ in $M 14$ is twice that in $M_{5}$. Considering the ratio size $(W / L)$ of the transistors, the above Eq. (3) can be written as,

$$
\begin{aligned}
& G_{m B D R F C} \\
& \quad \simeq\left[\left(\eta \cdot \sqrt{\frac{K_{P} \cdot(W / L)_{1 b}}{K_{N} \cdot(W / L)_{5}}}+1\right) \cdot(K+1)+\eta \cdot \sqrt{\frac{2 K_{P} \cdot(W / L)_{14}}{K_{N} \cdot(W / L)_{5}}}\right] \cdot g_{m 1 b}
\end{aligned}
$$

From Eq. (4), it can be seen that the enhancement factor $(\alpha)$ is described as follow,

$$
\alpha \simeq\left(\eta \cdot \sqrt{\frac{K_{P} \cdot(W / L)_{1 b}}{K_{N} \cdot(W / L)_{5}}}+1\right)+\frac{1}{K+1} \cdot \eta \cdot \sqrt{\frac{2 K_{P} \cdot(W / L)_{14}}{K_{N} \cdot(W / L)_{5}}}
$$

Assuming that $\eta \approx 0.3, K_{N} \approx 2 \cdot K_{P},(W / L)_{1 b}=4 \cdot(W / L)_{5}$ and $(W / L)_{14}=8 \cdot(W / L)_{5}$, thereby the enhancement factor $\alpha$ is equal to 1.6. Thus, compared to the conventional RFC, the proposed BDRFC using the proposed bulk-driven technique theoretically increases the effective transconductance almost $60 \%$. Also, the dc gain is enhanced almost $6 \mathrm{~dB}$ because of the enhanced transconductance.

\subsection{Common-mode range extended}

The threshold voltage of a MOS transistor as a function of the bulk-source voltage $V_{B S}$ is given by,

$$
V_{t h}=V_{t h 0}+\gamma\left(\sqrt{\left|2 \phi_{F}-V_{B S}\right|}-\sqrt{\left|2 \phi_{F}\right|}\right)
$$

where $V_{t h 0}$ is zero bias threshold voltage, $\gamma$ is bulk effect factor and $\phi_{F}$ is Fermi potential. The bulk bias $V_{B S}$ is normally $>0 V$, which numerically increases the threshold voltage. However, in the bulk-biasing technique, by biasing $V_{B S}<0 V$, the threshold voltage can be actually decreased. In the Fig. 2, the transconductance $\left(g_{m}\right)$ of transistor $M 1 a$ is given by,

$$
g_{m 1 a}=\frac{2 \cdot I_{B 1 a}}{V_{G S 1 a}-V_{T H 1 a}}
$$




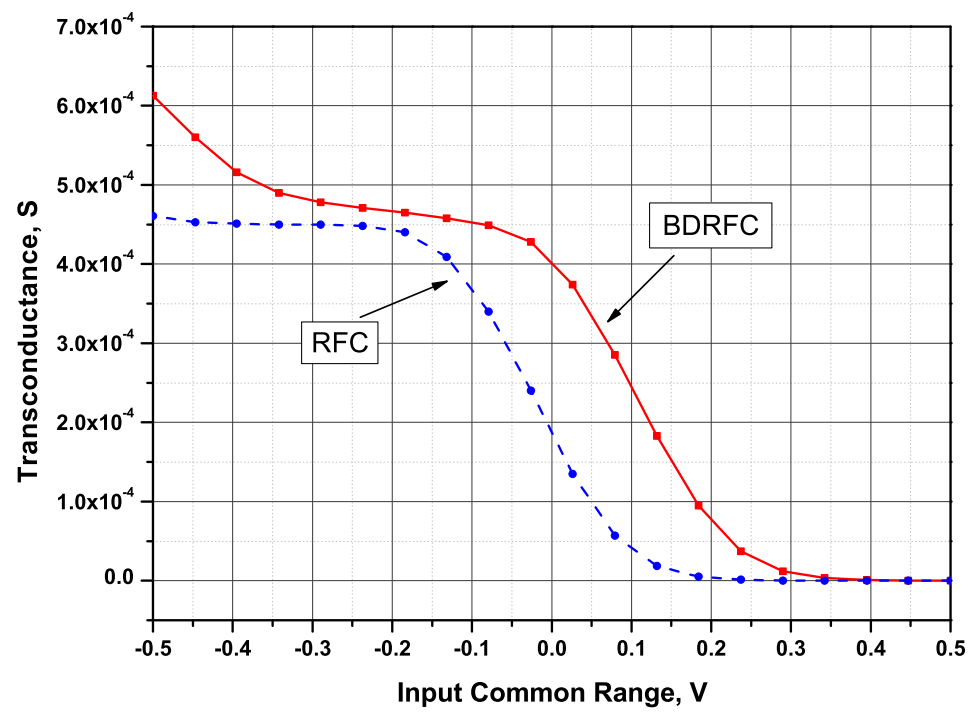

Fig. 3. The effective transconductance $G_{m 1 a}$ versus the input common-mode voltage range

as analyzed above, by applying a bias voltage $V_{A}$ to the bulk of $M 1 a$, the threshold voltage $V_{T H 1 a}$ is decreased because its bulk-source voltage becomes greater than zero. Decreasing $V_{T H 1 a}$ decreases its gate-source voltage $V_{G S 1 a}$, which leads to the input common-mode voltage range is extended. At the same time, $g_{m b 1 a}$ is equal to $\eta g_{m 1 a}$, where $\eta$ depends on $V_{B S}$ and other specific process parameters. In Fig. 3 the simulated effective $G_{m 1 a}\left(g_{m 1 a}+g_{m b 1 a}\right)$ as a function of the input common-mode voltage range is shown for the RFC and the BDRFC. It can be seen that the input common-mode voltage range of the BDRFC is extended when compared to the RFC.

In the proposed BDRFC, the bulk of both the input pairs and current mirror load are connected to the nodes $\mathrm{A}$ and $\mathrm{B}$, respectively. The dc level of the nodes A and B is $V_{t h n}+V_{o v}$ beyond the $V S S$, where $V_{o v}$ is overdive voltage, and it is approximately $500 \mathrm{mV}$ in $0.18 \mu \mathrm{m}$ process. Thus, in $1-\mathrm{V}$ supply voltage, the $V_{B S}$ of the input pairs $M_{i a, b}(i=1,2)$ and current mirror $M 13$ : $M 14$ will not forward bias the bulk-source diode.

\subsection{The stability performance}

The frequency performance of the BDRFC has been influenced and the phasemargin of the BDRFC is degraded. The dominant pole of the proposed BDRFC is determined by the load capacitance as the conventional RFC counterpart. However, the first non-dominant pole of the BDRFC is lowered owing to the parasitic capacitance of the bulk terminal.

In the RFC, the first non-dominant pole is expressed as,

$$
p_{A} \simeq \frac{g_{m 5}}{C_{g s 5}}
$$

where $C_{g s}$ is the gate-to-source capacitance. But the first non-dominant pole of the BDRFC is changed as,

$$
p_{A}^{*} \simeq \frac{g_{m 5}}{C_{g s 5}+C_{b s 2 a, b}+C_{b s u b 2 a, b}+C_{b s 14}+C_{b s u b 14}}
$$


where $C_{b s}$ is the bulk-to-source capacitance, and $C_{b s u b}$ is the well-to-substrate capacitance. From Eq. (9), it can be seen that the node A/B capacitance of the BDRFC is increased owing to the bulk terminal. Thus, the first nondominant pole is lowered, leading to the degradation of the phase-margin.

\section{Simulation results and discussion}

To demonstrate the performance enhancement, the two amplifiers, RFC and BDRFC, was simulated using CSMC standard $0.18 \mu \mathrm{m}$ CMOS process. Both of the OTAs consume a total current of $200 \mu \mathrm{A}$, using a supply voltage of $1-\mathrm{V}$, and deriving a capacitive load of $20.0 \mathrm{pF}$. The detailed aspect ratio of main transistors of the BDRFC are given in Table I.

Fig. 4 shows the simulated open-loop AC response of the RFC and BDRFC. Note that the dc gain is $72.8 \mathrm{~dB}$ for RFC and $78.7 \mathrm{~dB}$ for BDRFC. The dc gain of the BRFC improved almost $6 \mathrm{~dB}$ over the RFC, which is due to the improved transconductance. Also, the GBW of the RFC and BRFC

Table I. Transistors aspect ratios of the BDRFC

\begin{tabular}{lclc}
\hline Transistors & $\mathrm{W} / \mathrm{L}(\mathrm{um} / \mathrm{um})$ & Transistors & $\mathrm{W} / \mathrm{L}(\mathrm{um} / \mathrm{um})$ \\
\hline$M 1 a, b$ & $32 / 0.5$ & $M 2 a, b$ & $32 / 0.5$ \\
$M 3, M 5$ & $6 / 0.5$ & $M 4, M 6$ & $18 / 0.5$ \\
$M 7, M 8$ & $6 / 0.18$ & $M 9, M 10$ & $32 / 0.5$ \\
$M 11, M 12$ & $64 / 0.5$ & $M 13, M 14$ & $12 / 0.18$ \\
\hline
\end{tabular}
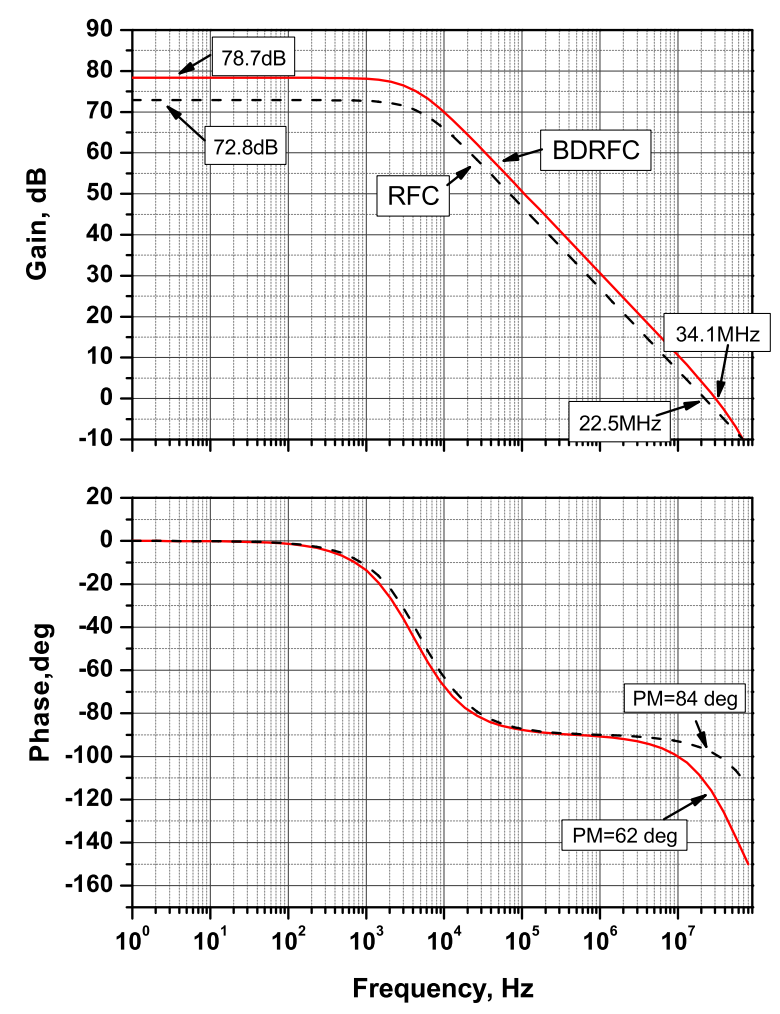

DOI: $10.1587 /$ elex.11.20131040 Received December 29, 2013 Accepted January 20, 2014 


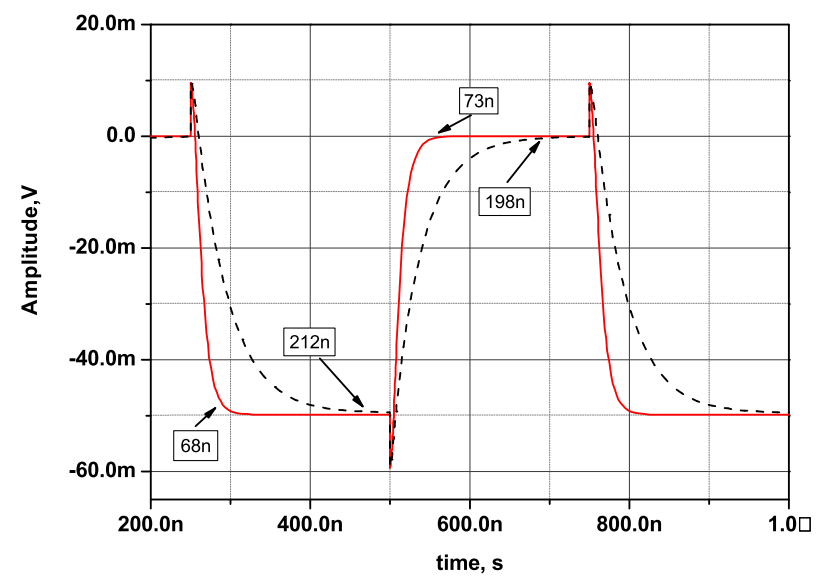

Fig. 5. The transient responses of the RFC and BDRFC

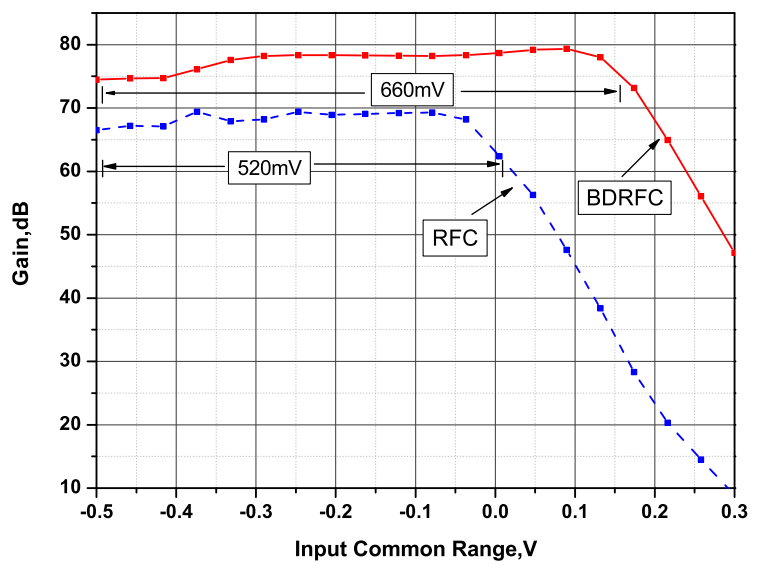

Fig. 6. The de gain versus the common-mode input voltage

is $22.5 \mathrm{MHz}$ and $34.1 \mathrm{MHz}$ respectively. The GBW improved $55 \%$ over that of the RFC, which indeed demonstrates the proposed bulk-driven technique. As for the phase margin, the BDRFC is degraded owing to the increased parasitic capacitive at node of the first non-dominant pole.

The simulation of transient responses to $2 \mathrm{MHz} 50 \mathrm{mVpp}$ step input are shown in Fig. 5. The settling time improvement of the BRFC is due to the higher GBW and the dc gain enhancement of BRFC leads to the reduced static error.

Fig. 6 shows that the de gain as a function of the common-mode input voltage in the RFC and the BDRFC. At $1-\mathrm{V}$ power supply, a $0.66 \mathrm{~V}$ commonmode input range in which the proposed $\mathrm{BDRFC}$ has at least a $73 \mathrm{~dB}$ gain. When compared to the RFC counterpart, it is extended by $140 \mathrm{mV}$.

Considering process variation, ac response of the proposed BDRFC has been done under different corners, which is shown in Fig. 7. It shows that the variation of gain-bandwidth is less than $1.8 \mathrm{MHz}$ and that of dc gain is less than $6.8 \mathrm{~dB}$.

A comparison between the proposed BDRFC and the traditional RFC is shown in Table II where both amplifiers have the same power dissipation and the same load capacitance. From the Table II, it can be seen that the 

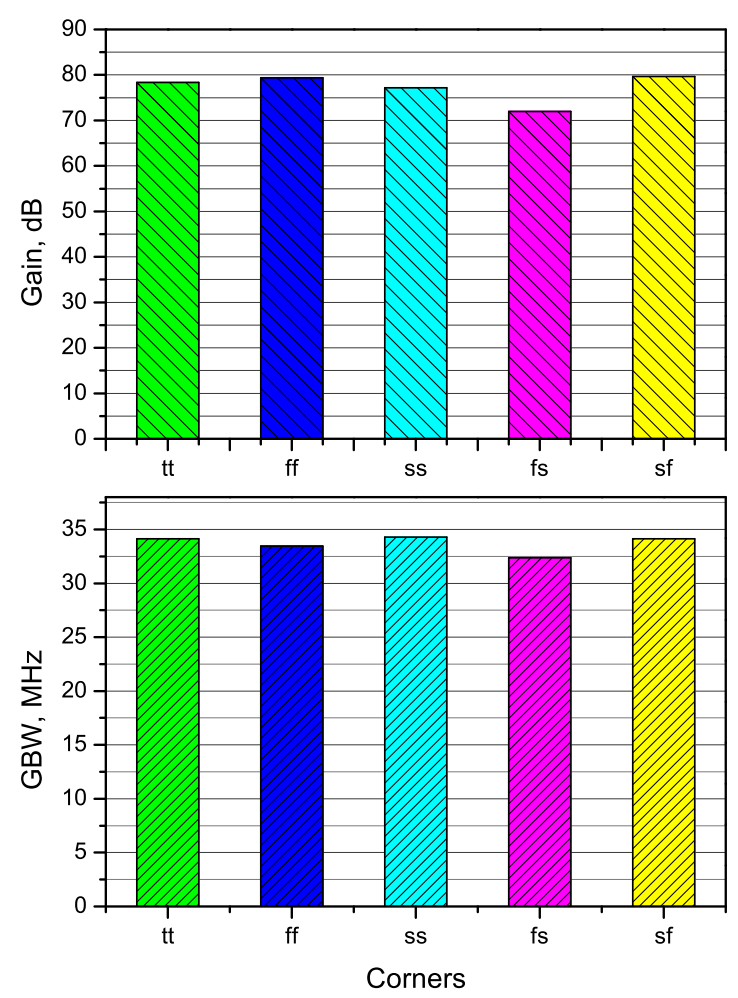

Fig. 7. DC gain and GBW of the BDRFC under different corners

Table II. Performance summary of RFC and BDRFC

\begin{tabular}{lcc}
\hline Parameter & RFC & BDRFC \\
\hline Technology & $0.18 \mathrm{um}$ & $0.18 \mathrm{um}$ \\
Supply Voltage $[\mathrm{V}]$ & 1 & 1 \\
Capacitive Load [pF] & 20 & 20 \\
Power (Bias current) [uA] & 200 & 200 \\
DC Gain [dB] & 72.8 & 78.7 \\
GBW [MHz] & 22.5 & 34.1 \\
Open Loop PM [deg] & 84 & 62 \\
ICMR (mV) & 520 & 660 \\
Slew rate (average) [V/us] & 1.8 & 4.2 \\
1\% settling time (average) [ns] & 205 & 65.5 \\
Output swing $(\mathrm{mV})$ & 620 & 730 \\
Input Noise $\left[n V / H z^{1 / 2}\right] @ 1 \mathrm{MHz}$ & 14.2 & 12.5 \\
FoM (MHzpF/uA) & 225 & 341 \\
\hline
\end{tabular}

FoM of the BDRFC is 341 while that of RFC is 225 , which has a significant enhancement. In addition, since the decreased threshold voltage of transistor $M 13$ and $M 14$, the output swing of the BDRFC increases a $110 \mathrm{mV}$ when compared to the RFC. 


\section{Conclusion}

A proposed bulk-driven recycling current folded cascode OTA (BDRFC) is presented. Under the CSMC $0.18 \mu \mathrm{m}$ CMOS process, two OTAs were designed. To compare the performance versus the conventional counterpart, the proposed OTA's unit-gain bandwidth is improved by $55 \%$ and the input/output voltage range is increased by $140 / 110 \mathrm{mV}$. Also, the dc gain is enhanced almost $6 \mathrm{~dB}$.

\section{Acknowledgments}

This work is supported by the National 863 Program of China (No.2012AA09A20102 and No.2012AA061102), the National Natural Science Foundation of China (No. 41204135), and the Fundamental Research Funds for the Central Universities of China (No. 2652011262). 